\title{
Transition from Islanded to Grid-Connected Mode of Microgrids with Voltage-Based Droop Control
}

\author{
T. L. Vandoorn, Student Member, IEEE, B. Meersman, Student Member, IEEE, J. D. M. De Kooning, Student Member, \\ IEEE and L. Vandevelde, Senior Member, IEEE
}

\begin{abstract}
Microgrids are able to provide a coordinated integration of the increasing share of distributed generation (DG) units in the network. The primary control of the DG units is generally performed by droop-based control algorithms that avoid communication. The voltage-based droop (VBD) control is developed for islanded low-voltage microgrids with a high share of renewable energy sources. With VBD control, both dispatchable and less-dispatchable units will contribute in the power sharing and balancing. The priority for power changes is automatically set dependent on the terminal voltages. In this way, the renewables change their output power in more extreme voltage conditions compared to the dispatchable units, hence, only when necessary for the reliability of the network. This facilitates the integration of renewable units and improves the reliability of the network. This paper focusses on modifying the VBD control strategy to enable a smooth transition between the islanded and the grid-connected mode of the microgrid. The VBD control can operate in both modes. Therefore, for islanding, no specific measures are required. To reconnect the microgrid to the utility network, the modified VBD control synchronises the voltage of a specified DG unit with the utility voltage. It is shown that this synchronisation procedure significantly limits the switching transient and enables a smooth mode transfer.
\end{abstract}

Index Terms-Distributed generation, droop control, microgrid, synchronisation

\section{INTRODUCTION}

The environmental goals and liberalisation of energy markets have led to a huge increase of distributed generation (DG) units (often with renewable energy sources) in the electrical power system. This has a large impact on the power system planning and operation [1]-[4]. With a high penetration of DG, the voltage and congestion problems can significantly reduce the hosting capacity of the networks for DG [5]. Also, as the ratio DG power versus centrally-generated power increases, the DG units will need to assist in ancillary services such as reserve provision. Therefore, the fit-and-forget strategy for integrating DG is not a sustainable option and a coordinated approach with active control of these units will be required.

With respect to the coordinated integration of DG, the microgrid concept has been developed. Microgrids are power systems that consist of an aggregation of loads, sources and storage elements [6], [7]. An important characteristic of the microgrid is that it is regarded as a controllable entity from the utility networks

This work is financially supported by the FWO-Vlaanderen (Research Foundation - Flanders, Belgium). T. Vandoorn thanks the FWO for the Fellowship received. The research was carried out in the frame of the Inter-university Attraction Poles programme IAP-VII-43, funded by the Belgian Government. The research of J. D. M. De Kooning is funded by the Special Research Fund (BOF) of Ghent University (Belgium).

T. L. Vandoorn, B. Meersman, J. D. M. De Kooning and L. Vandevelde are with the Electrical Energy Laboratory (EELAB), Department of Electrical Energy, Systems and Automation (EESA), Ghent University, Sint-Pietersnieuwstraat 41, B-9000 Ghent, Belgium, e-mail: Tine.Vandoorn@UGent.be point of view. This is made possible because the microgrid has a single point of common coupling (PCC). Dependent on the state of the PCC switch, the microgrid can operate in grid-connected and islanded mode. Microgrids are likely to play a key role in the evolution of the smart grid [8], [9]. It is expected that the smart grid will emerge as a system of integrated smart microgrids [10]. As most DG units are connected to the network through a voltagesource inverter (VSI), proper microgrid operation requires proper inverter control.

The inverter control of the DG units in grid-connected microgrids is related to the delivery of a certain amount of power to the network. Generally, grid-following units, with current controllers that track the measured terminal voltage, are used [11]-[13]. The grid stability and power quality remain a task of the transmission system. In the islanded mode, the inverter controllers are responsible for the microgrid stability and power quality. Gridforming, thus voltage-controlled, units are required because of the lack of a utility network forming the reference voltage. Generally, droop-based control algorithms are used for the primary control in islanded microgrids, to avoid communication and single points of failure for a reliable system operation.

Droop control can be classified in $P / f$ and $P / V_{\mathrm{g}}$ droops, with $P$ the active power, $f$ the grid frequency and $V_{\mathrm{g}}$ the terminal rms voltage. The former focusses on mimicking the $P / f$ controllers of the synchronous generators connected to the transmission networks [7], [14]-[16]. Low-voltage microgrid are considered in this paper, which generally lack the rotating inertia the conventional grid control is based upon. Also, the lines are predominantly resistive such that there is a linkage between $P$ and $V_{\mathrm{g}}$, not frequency (through phase angles). Therefore, $P / V_{\mathrm{g}}$ droops have been used in [17], [18]. The $P / V_{\mathrm{g}}$ droop controller focusses on dispatchable DG units. A variant of these $P / V_{\mathrm{g}}$ droops, called the voltage-based droop (VBD) control, has been presented in [19]. This control strategy takes into account the less dispatchable nature of various DG units. It also enables to use the voltage as trigger for primary load and storage control in [20].

An important benefit of the VBD control strategy is that it can be used in both the grid-connected and the islanded mode of the microgrid. Hence, in the event of islanding, i.e., transition from grid-connected to islanded mode, the microgrid will remain online without changing the control strategy. During the transition from islanded to grid-connected mode on the other hand, synchronisation of the microgrid voltage to the utility voltage is required. Otherwise, large switching transients in voltage and current may occur. Hence, in this paper, a synchronisation procedure is included in the VBD controller, while retaining the same control strategy in the grid-connected and islanded mode. In [21], synchronisation of a DG unit with a modified control strategy in both modes is presented. In [22]-[24], the synchronisation of a multiple DG microgrid is achieved by 
aligning the voltage phasors at the microgrid and utility ends. Two synchronisation compensators are included, one for voltage magnitude compensation through a modified $Q / V$ controller and a second to eliminate phase errors through the modified $P / f$ controllers. For the VBD control, the voltage magnitude synchronisation is achieved through the active power controller, phase synchronisation is obtained by reactive power changes. Hence, the synchronisation procedure is reversed compared to the conventional synchronisation.

This paper studies the mode transition of a microgrid by including a synchronisation procedure in the VBD control strategy. Hence, the VBD control principle is discussed with respect to operation in the islanded mode ( $\$$. II.A) and the grid-connected mode (§. II.B). Next, the transition from grid-connected to islanded mode (§. II.C) and vice versa (§. II.D) are analysed, emphasizing that the latter transition requires a synchronisation procedure. This paragraph also discusses the changes required in the VBD controller to enable the synchronisation. $§$ III, some cases are studied to verify that, in a basic and an extended microgrid: (1) VBD control is possible in both operating modes; (2) islanding does not require additional control modifications; (3) the synchronisation procedure leads to a smooth reconnection of the microgrid to the utility network.

\section{Control Strategies}

In this paragraph, the primary control of the DG units in a microgrid is considered. The control algorithm of the VSI of the DG units is based on a droop concept, thus, without master DG unit in the grid-connected and islanded operating condition. One unit is responsible for the synchronisation. All DG units are power-electronically interfaced and the microgrid has multiple points of DG connection as shown in Fig. 1 .

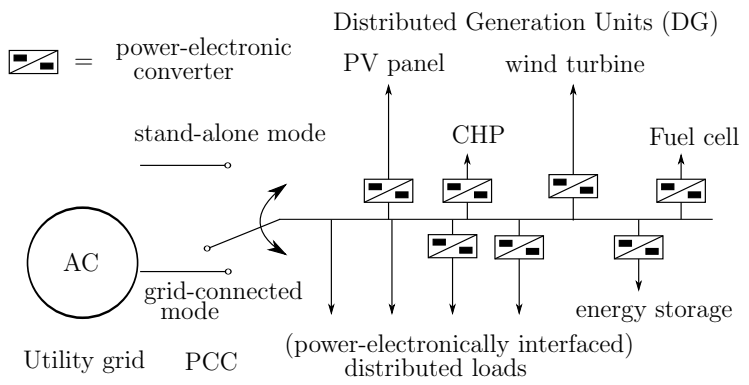

Fig. 1. Microgrid with (power-electronically interfaced) loads, storage and DG units in stand-alone or grid-connected mode

\section{A. VBD control in islanded microgrids}

In islanded networks, at least one grid-forming DG unit is required that is voltage controlled. The VBD control scheme sets the reference voltage of the DG units' voltage controllers to enable power sharing and balancing.

1) Voltage control: For the voltage controller, conventional inner current and outer voltage PI controllers are used: [25], [26]. In the converter, pulse width modulation with sampling period $T_{\mathrm{b}}$ is used, leading to discrete reference values for the voltage control block. The reference value $v_{\mathrm{k}}^{\star}=V_{\mathrm{g}, \mathrm{k}} \sin \left(\alpha_{\mathrm{k}}\right)$ is obtained from $V_{\mathrm{g}}\left(V_{\mathrm{g}} / V_{\mathrm{dc}}\right.$ droop controller) and $f(Q / f$ droop controller $)$ at the discrete instant $k$

$$
v_{\mathrm{k}}^{\star}=V_{\mathrm{g}, \mathrm{k}} \sin \left(\alpha_{\mathrm{k}-1}+2 \pi f_{\mathrm{k}} T_{b}\right) .
$$

These droop controllers are discussed below.
2) Active power control: The active power controller of the VBD control is based on two control strategies, with their operation dependent on the rms microgrid voltage $V_{\mathrm{g}}$ [19].

In a voltage band around the nominal voltage, only the $V_{\mathrm{g}} / V_{\mathrm{dc}}$ droop control strategy is applied, with $V_{\mathrm{dc}}$ the dc-link voltage of the power source and $K_{\mathrm{V}}$ the droop coefficient:

$$
V_{\mathrm{g}}=V_{\mathrm{g}, \mathrm{nom}}+K_{\mathrm{V}}\left(V_{\mathrm{dc}}-V_{\mathrm{dc}, \text { nom }}\right) \text {. }
$$

For power-electronically interfaced DG units, a difference between the ac power $P$ and the input power $P_{\mathrm{dc}}$ is reflected in a changing $V_{\mathrm{dc}}$. To limit this dc-voltage change, the $V_{\mathrm{g}} / V_{\mathrm{dc}}$ droop controller affects $P$ by changing $V_{\mathrm{g}}$ proportional to $V_{\mathrm{dc}}$ [19]. This controller is based on the natural linkage between $P$ and $V_{\mathrm{g}}$ in the considered microgrids, which are mainly resistive [18], [27].

However, the ac-voltage needs to be limited as well. Therefore, if the microgrid voltage exceeds the aforementioned voltage band, also a $P_{\mathrm{dc}} / V_{\mathrm{g}}$ droop controller is turned on as shown in Fig. 2 This controller changes the generated power $P_{\mathrm{dc}}$ and avoids voltage limit violation:

$$
P_{\mathrm{dc}}=\left\{\begin{array}{l}
P_{\mathrm{dc}, \text { nom }}-K_{\mathrm{p}}\left(V_{\mathrm{g}}-(1+b) V_{\mathrm{g}, \text { nom }}\right) \\
\quad \text { if } V_{\mathrm{g}}>(1+b) V_{\mathrm{g}, \text { nom }} \\
P_{\mathrm{dc}, \text { nom }} \\
\quad \text { if }(1-b) V_{\mathrm{g}, \text { nom }}<V_{\mathrm{g}}<(1+b) V_{\mathrm{g}, \text { nom }} \\
P_{\mathrm{dc}, \text { nom }}-K_{\mathrm{p}}\left(V_{\mathrm{g}}-(1-b) V_{\mathrm{g}, \text { nom }}\right) \\
\quad \text { if } V_{\mathrm{g}}<(1-b) V_{\mathrm{g}, \text { nom }}
\end{array}\right.
$$

with $K_{\mathrm{p}}$ the droop coefficient and $b$ called the constant-power band. In the dispatchable units, $P_{\mathrm{dc}, \text { nom }}$ is generally the scheduled power of the unit, e.g., in the day-ahead markets, thus, varies over time. This value is highly dependent on the ratings of the unit, which corresponds to the operating point with optimal efficiency. For the renewable DG units, $P_{\mathrm{dc}, \text { nom }}$ is generally the Maximum Power Point (MPP). This value will, hence, also vary in time. Fig. 2 shows the VBD control of the DG unit's VSI. In a PV panel, for example, this inverter's dc-side is connected to a capacitor, which is connected with the PV panel through a dc-dc converter, e.g., a boost chopper. VBD control focusses on the acside inverter, hence, $P_{\mathrm{dc}, \text { nom }}$ is a given value from this controller's point of view as it is the output of a MPP tracking algorithm of the dc-dc converter. In literature, several MPPT algorithms have been presented, such as the hill climbing algorithms, look up table method and incremental conductance for PV [28]-[30]. This paper focusses on the VSI control, not the dc-dc converter control.

Both droop controllers, (2) and (3), do not depend on communication and deal with the specific properties of the microgrid, such as the lack of inertia, the resistive line characteristics and the (transient) storage capabilities of the dc-link capacitors.

The $P_{\mathrm{dc}} / V_{\mathrm{g}}$ droop controller operates if a certain threshold microgrid voltage is exceeded as shown in Fig. 2. The upper and lower threshold voltages $(1 \pm b) V_{\mathrm{g}, \text { nom depend on the }}$ characteristics of the power source, which is illustrated in Fig. 3 For example, for variable, controlled (often non-renewable) power sources, a narrow constant-power band is handled. Therefore, small variations of $V_{\mathrm{g}}$ from $V_{\mathrm{g} \text {,nom }}$ address the $P_{\mathrm{dc}} / V_{\mathrm{g}}$ droop controller to change $P_{\mathrm{dc}}$. This enables to fully exploit the power control characteristics of the dispatchable power sources. In this way, less voltage variation is obtained in the microgrid as the power sources act dynamically to limit the voltage changes by 
changing their output power. For non-variable or slightly-variable power sources (often intermittent renewable or combined heat and power units with heat as primary driver), $P_{\mathrm{dc}}$ is determined externally and therefore, a wide constant-power band should be applied. In this way, changing the output power of the less dispatchable power sources is only addressed to limit large voltage variations, ensuring a reliable microgrid operation. Because of the increasing share of renewable energy sources, active dispatching of these units in small-scale microgrids will be required, e.g. to avoid over-voltage tripping. This control strategy makes this possible, while still delaying the power changes of the renewables to more extreme voltages compared to those of the dispatchable DG units.

In conclusion, by setting the value $b$, the priority in which the units react on load variations is set, dependent on variations of the voltage from its nominal value. For small variations, the dispatchable DG units and storage elements (small b) will react. Only for more extreme voltages, the other units, such as controllable loads or renewables will react as well. Fig. 3 (b) represents for example a combination of a renewable energy source and a controllable load. The power can decrease by the renewable source through deviation from the maximum power point, and a power increase is equivalent with a load decrease (load shifting). If only the renewable unit is considered, the droop characteristic can be changed following Fig. 3. (c).

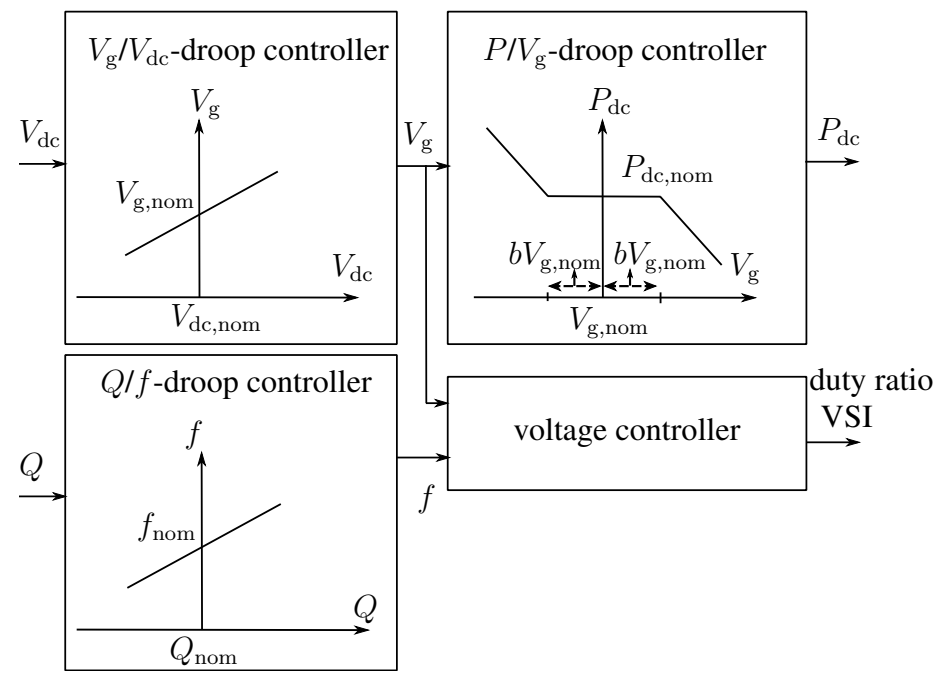

Fig. 2. Combined operation of the droop controllers to determine the set value of the grid voltage in case of VBD control

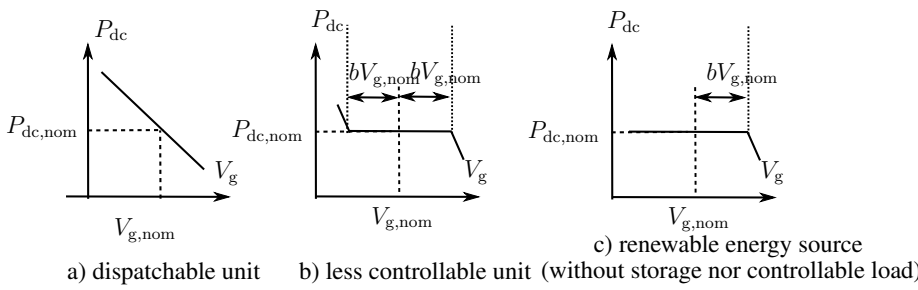

Fig. 3. Fully dispatchable versus fully undispatchable DG unit. Dispatchable units have a small constant power band $(2 b=0 \%)$, fully undispatchable DG units have a wide constant-power band.
3) Reactive power control: In the considered low-voltage microgrids, the reactive power $Q$ is mainly linked with phase angle differences, because the lines are mainly resistive. Therefore, a $Q / f$ droop controller is applied for the reactive power sharing between the DG units:

$$
f=f_{\text {nom }}+K_{\mathrm{Q}}\left(Q-Q_{\text {nom }}\right),
$$

with $K_{\mathrm{Q}}$ the droop coefficient. The reactive power sharing between the different generators can be influenced by adjusting the droops.

\section{B. VBD control in grid-connected microgrids}

Although developed for islanded microgrids, the VBD controller can be used in grid-connected networks as well.

The mains frequency, which is determined by the droop controllers of the large conventional generators, will be little affected by the small grid-connected microgrid. Hence, the frequency of the microgrid units $\left(f_{\mathrm{dg}}\right)$ will converge to the mains frequency $\left(f_{\text {mains }}\right)$. For example, if $f_{\mathrm{dg}}>f_{\text {mains }}$, the phase angle of the microgrid units ( $\delta$ for a specific unit) will keep increasing compared to that of the PCC $\left(\delta_{\text {mains }}=0\right)$. From the power flow equations of a DG unit to the utility in a resistive network:

$$
Q \approx \frac{-V_{\mathrm{dg}} V_{\text {net }}}{R_{\text {line }}} \delta,
$$

it follows that the reactive power delivered by this unit to the utility will decrease. The DG unit measures its $Q$ and droops it with a positive slope, which in turn leads to a lower set point of frequency $f_{\mathrm{dg}}$. In this way, a steady-state is reached. Note that because of the small and temporarily differences between the frequencies, this problem should not be analysed in terms of frequency differences, but in terms of phase angle differences, as discussed above. In general, $f_{\text {mains }} \approx f_{\text {nom }}$, such that the reactive power of the unit will approximately equal its nominal value. If $Q_{\text {nom }}=0 \mathrm{VAr}$ in the VBD control, this is equal to the conventional control strategies of DG units with power-factorone.

Like in the islanded microgrid, the $V_{\mathrm{g}} / V_{\mathrm{dc}}$ droop controller takes care of the balancing of the dc-bus. The $P_{\mathrm{dc}} / V_{\mathrm{g}}$ droop controller changes the active power of the unit to enable voltage limiting in the network. Opposed to the widely-used on/off control of renewables, the VBD control uses soft curtailment to temporarily lower the output power in case of over-voltage, analogous to [5]. The constant-power band enables to only use curtailment to ensure a reliable operation. Hence, the renewables will generally operate at maximum power point, which is analogous as in the conventional DG control. The other units, with smaller constant-power bands, will change their output power dependent on the network voltage. This is beneficial to avoid voltage limit violation, but can be overruled by changing the droops, constant-power bands or nominal values in an overlaying slower control.

The VBD control is a primary control, operating very fast to ensure a reliable microgrid operation. It can be included in a hierarchical control algorithm. Secondary control can deal with economic issues and can restore the nominal values of rms voltage and frequency, by changing the set points of the primary controllers. This is out of the scope of this paper; details concerning hierarchical control of microgrids are given in [25]. 


\section{Transition from grid-connected to islanded mode}

When considering the transition from the grid-connected to islanded mode, two types of islanding are possible: planned and unplanned. Both types generally do not pose problems for the microgrid when using the VBD control. The control strategy can remain the same in both modes. In grid-connected mode, the terminal voltage and frequency of the units are generally near their nominal values. Hence, in this mode, the units deliver their nominal active and reactive power, analogous as in the gridfollowing strategies. Consequently, the small DG units do not contribute in the power sharing as they deliver power independent on the state of the network, e.g., renewables deliver the maximum available power. If all the DG units would keep on delivering their nominal power to the network in islanded mode, this would lead to large variations of the voltage compared to its nominal value. Hence, the VBD controller acts on these deviations, limits them and takes care of the power balancing and power sharing.

As the VBD control strategy can remain the same in the two modes, obviously, for islanding, no synchronisation is required. This is not valid when instead of VBD control, a grid-following control strategy is used. In this case, a change of control strategy is required as the islanded mode demands for grid-forming control strategies for the voltage control, power balancing and power sharing in the network.

\section{Transition from islanded to grid-connected mode}

Opposed to islanding, the transition from islanded to gridconnected mode is generally planned. Before the transition, the phase angle and rms value of the PCC voltage at microgrid and utility side can differ. Moreover, the microgrid and utility network can operate at a different frequency, because the droop controllers in both networks do not force the frequency to its nominal value. Therefore, closure of the PCC switch without synchronisation would lead to large transients, i.e. sudden voltage and current changes. In order to realize a smooth mode transfer, a synchronisation procedure is required. Therefore, in this paper, the VBD control strategy is changed to synchronise the utility and microgrid side rms voltage, phase angle and frequency before connecting the microgrid to the utility.

The synchronisation procedure can operate slower compared to the primary (VBD) control. Therefore, it is not necessary to avoid communication. The PCC voltage is measured and communicated to the synchronising DG unit that uses a phase-locked loop (PLL) to obtain the rms voltage and its phase angle. One unit is selected for the synchronisation of the microgrid. To cope with this responsibility, this unit should be sufficiently large, dispatchable and close to the PCC.

The synchronisation strategy to synchronise the DG unit's voltage $v_{\mathrm{g}}$ to the PCC voltage at the utility side $v_{\text {util }}$ is illustrated in Fig. 4 As the synchronising unit is electrically close to the PCC, it is assumed that $v_{\mathrm{g}} \approx v_{\mathrm{PCC}}$. The PCC switch closes at $t=t_{\text {conn }}$ and the synchronisation procedure is active in $t_{\text {conn }}-T_{\text {sync }}<t<t_{\text {conn }}$, with $T_{\text {sync }}$ the duration of the synchronisation procedure. As discussed above, the VBD controller consists of a dc-power controller with the input coming from the $P_{\mathrm{dc}} / V_{\mathrm{g}}$ droop controller and a voltage controller with input from the $V_{\mathrm{g}} / V_{\mathrm{dc}}$ and $Q / f$ droop controllers. In this paper, the VBD controller of the synchronising unit is modified by including an rms voltage synchronisation block, a droop limiting block and a phase synchronisation block. The voltage, phase angle and frequency of the synchronising DG unit are controlled to their respective PCC values: $V_{\mathrm{g}}=V_{\text {util }}, \theta_{\mathrm{g}}=\theta_{\text {util }}$ and $f=f_{\text {util }}$. These three synchronisation tasks are performed by the three different control blocks.

The rms voltage synchronisation block changes the output of the $P_{\mathrm{dc}} / V_{\mathrm{g}}$ droop controller gradually, thus, by using a ramp function, to force $V_{\mathrm{g}}$ to $V_{\mathrm{util}}$.

Phase angle synchronisation is enabled by the phase synchronisation block that control $\theta_{\mathrm{g}}$ towards $\theta_{\text {util }}$. Again, a gradual synchronisation is obtained by using a ramp function. The output of this block is a frequency change, which according to (1) changes the phase angle of the voltage reference.

The DG unit's frequency is controlled to the grid frequency by using the droop limiting block. This block turns off the $Q / f$ droop ( $f=f_{\text {nom }}$ ) sufficiently before the synchronisation is performed. In Fig. 4 the gain of the droop is gradually lowered until it is off at $t=t_{\text {conn }}-T_{\text {sync }} / 2$. Here, it is assumed that the grid frequency equals $50 \mathrm{~Hz}$, the same strategy can be used for variable grid frequency, with the difference that $f_{\text {nom }}$ in Fig. 4 is changed to $f_{\text {util }}$ during synchronisation.
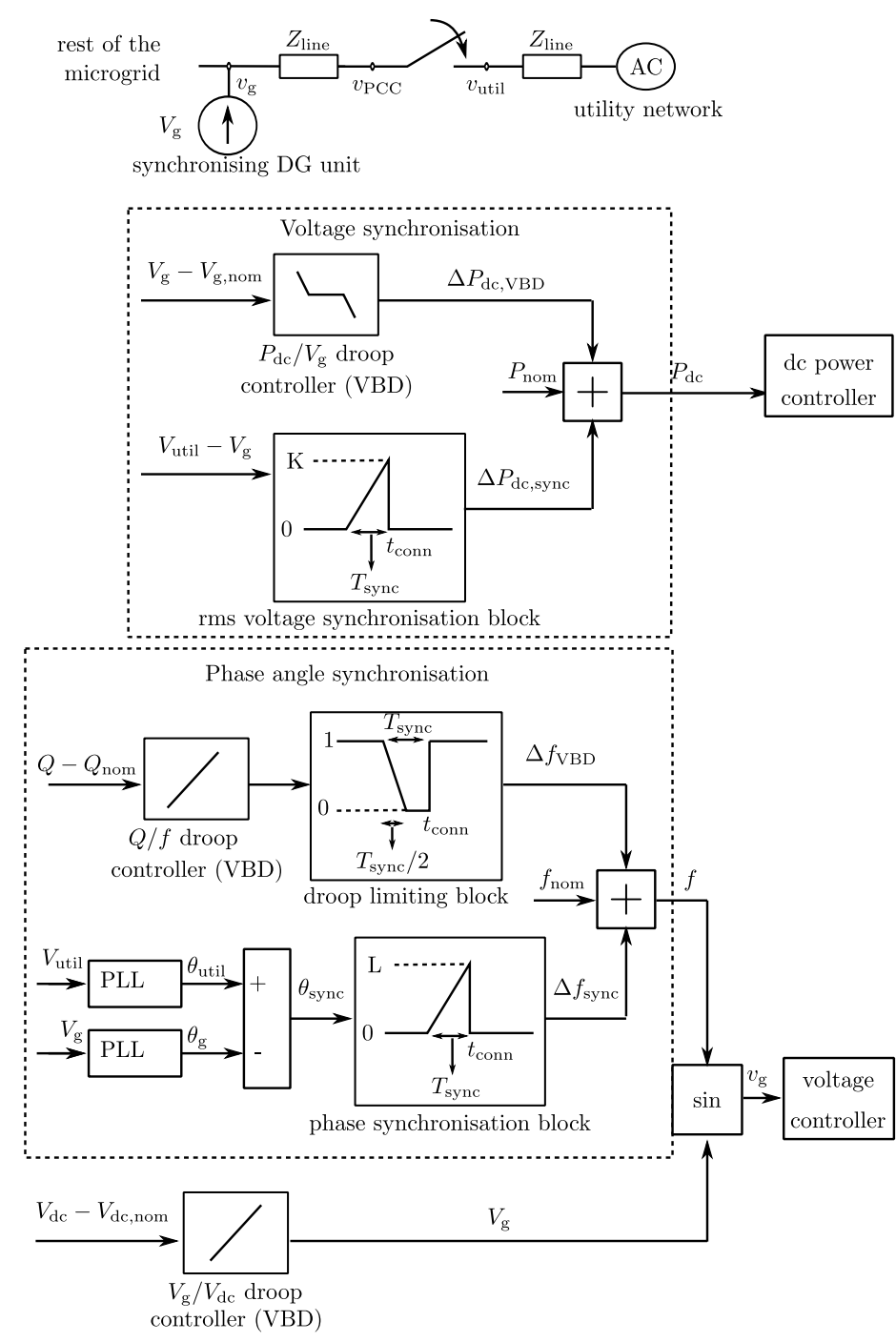

Fig. 4. Synchronisation: control strategy in the VBD control, with $V_{\mathrm{g}}$ the rms value of the DG unit's terminal voltage $v_{\mathrm{g}}(\mathrm{t})$ 


\section{MICROGRID MODE TRANSFER WITH VBD CONTROL: RESULTS}

A basic and extended microgrid with mode transition are studied. First, the islanding procedure is discussed. Second, the transition from islanded to the grid-connected mode is discussed, and a comparison is made between the cases with and without synchronisation. Matlab Simulink with the Plecs library, to model the inverters upto the switches level, is used. It is shown that without synchronisation, large transients in voltage and current can occur and that the presented synchronisation procedure is effective to enable a smooth mode transition.

\section{A. Basic microgrid}

The considered microgrid, with two DG units and two RL loads, is depicted in Fig. 5. The utility network is modelled as a voltage source of $230 \mathrm{~V}$ rms and $50 \mathrm{~Hz}$, i.e., a strong network connected to the microgrid through a line impedance $(R / X=1)$. A large parasitic resistance is present in parallel with the inductance of this line. The parameters of the DG units are: $L=2 \mathrm{mH}$, $C=3 \mu \mathrm{F}, C_{\mathrm{dc}}=1.5 \mathrm{mF}$. The nominal values are: $V_{\mathrm{dc}, \text { nom }}=$ $450 \mathrm{~V}, P_{\mathrm{dc}, \text { nom }, 1}=1.6 \mathrm{~kW}, P_{\mathrm{dc}, \text { nom }, 2}=800 \mathrm{~W}, Q_{\mathrm{dc}, \text { nom }, 1}=$ $Q_{\mathrm{dc}, \text { nom }, 2}=0$ VAr and the constant-power band $b$ equals $1 \%$. As a low-voltage microgrid is considered, the lines in the microgrid are resistive, here $0.3 \Omega$ [18], [27].

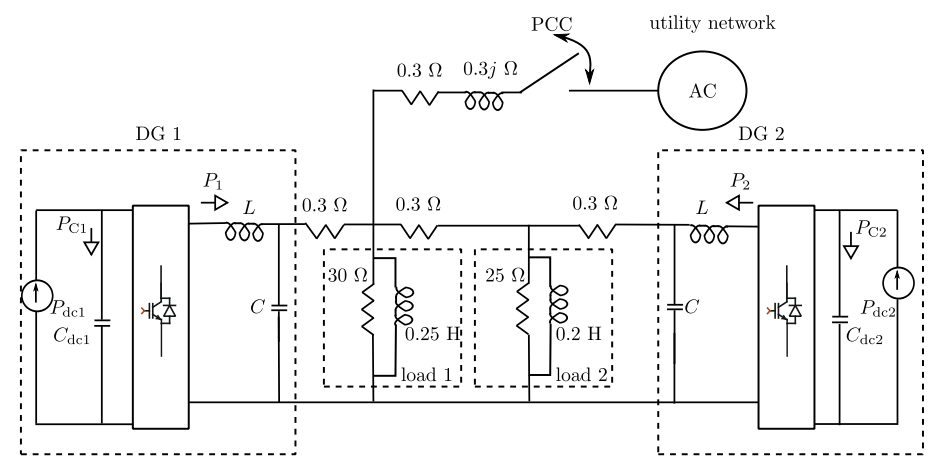

Fig. 5. Basic microgrid configuration

1) VBD control with transition from grid-connected to islanded mode: First, the transition from grid-connected to islanded mode, i.e., islanding, is considered. The transition takes place at $t=$ $0.505 \mathrm{~s}$.

The obtained results in Fig. 6 show an adequate transition, with limited switching transients. The DG units operate with VBD control in both modes. The voltage remains in the $10 \%$ limits. The obtained voltage drop after switching is relatively large due to the small scale of the microgrid studied here, the lack of storage equipment and the large load burden. Here, only primary control (stabilization of the microgrid) is considered. If required, an overlaying secondary controller will change the set points of the primary controller to force the voltage closer to the nominal value, enabling voltage restoration on a longer term. This is out of the scope of this paper.

The active power of both units is shared according to their ratings and droops. In steady state for the islanded operation, DG 1 delivers $2.2 \mathrm{~kW}$, while DG 2 delivers $1.1 \mathrm{~kW}$. In both modes, the DG units contribute in the voltage control because of their small constant-power band as they represent dispatchable units. Therefore, the active power differs from its nominal value.

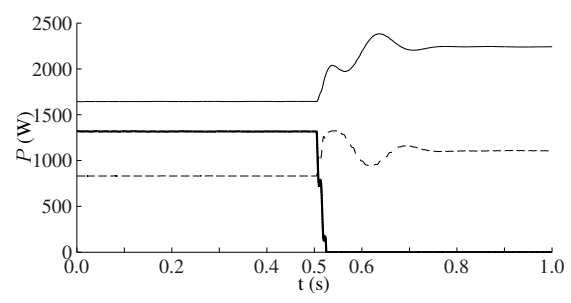

(a) Delivered active power

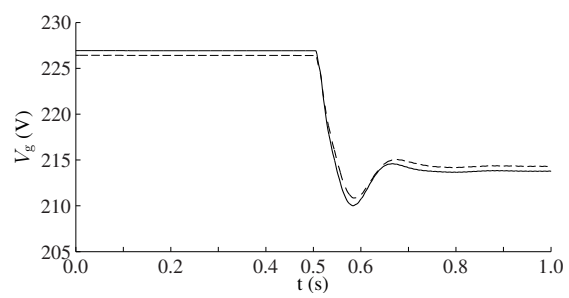

(b) Terminal rms voltage

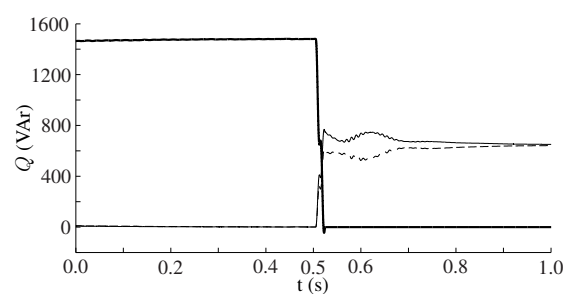

(c) Delivered reactive power

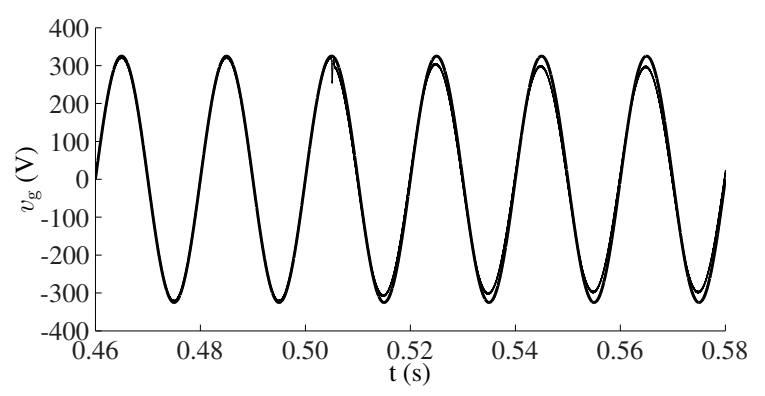

(d) Terminal voltage

Fig. 6. Basic microgrid: grid-connected to islanded (- = DG 1; ---- = DG 2; $-=$ utility network)

This enables soft curtailment by the VBD control in a primary controller. However, as in grid connected mode $V_{\mathrm{g}} \approx 230 \mathrm{~V}$, the DG units nearly deliver their rated power, $P_{1}=1.60 \mathrm{~kW}$ and $P_{2}=0.83 \mathrm{~kW}$. Note that less-dispatchable DG units, thus, with a wider constant-power band, would exactly deliver their nominal power, i.e., maximum power point, in this case. In the grid-connected mode, the DG units operate with power-factor-one as $f=f_{\text {nom }}$ because of the strong utility network. In islanded mode, the reactive power of the loads is shared between the DG units. Here, the units deliver equal reactive power because of the equal droop and equal nominal values of $f$ and $Q$ chosen in this case.

Fig. 6.d) shows that in the grid-connected mode, the voltage of DG 1 , which is electrically close to the PCC, differs little from the utility's voltage. After islanding, the difference becomes larger because of the droop controllers that enable power balancing and power sharing.

The simulations show a small islanding transient and a proper 


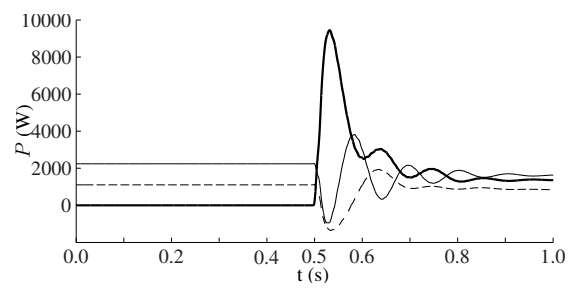

(a) Delivered active power

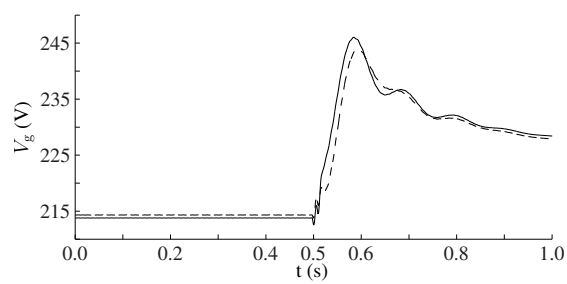

(b) Terminal rms voltage

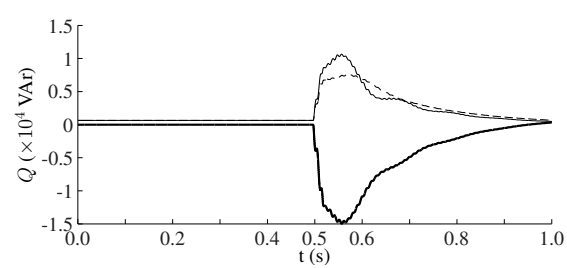

(c) Delivered reactive power

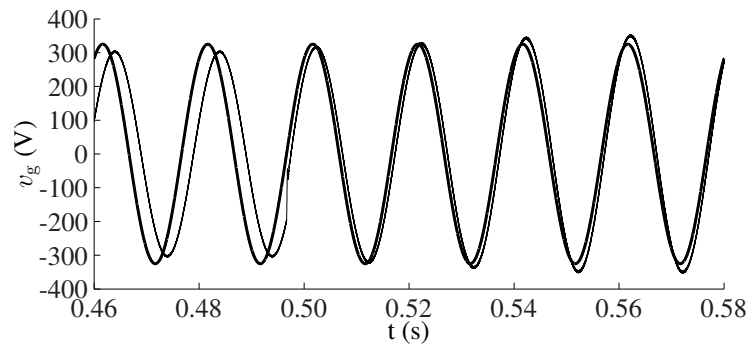

(d) Terminal voltage

Fig. 7. Basic microgrid: islanded to grid-connected, no synchronisation $(-=$ DG $1 ;----=$ DG $2 ;-=$ utility network)

operation of the VBD controller in both modes.

2) VBD control with transition from islanded to grid-connected mode: The voltage $v_{\mathrm{util}}(\mathrm{t})$ is shifted with $\pi / 3$ relative to $v_{\mathrm{g}}(\mathrm{t})$. In this way, synchronisation of $v_{\mathrm{g}}$ and $v_{\mathrm{util}}$ is required. The transition occurs at the zero-crossing of $v_{\text {util }}$, just before $t=0.5 \mathrm{~s}$.

a) Without synchronisation procedure: In the first case, the DG units are not synchronized to the utility network, which is depicted in Fig. 7(d). Figs. 7(a-c) show extreme transients in $P$, $Q$ and $V_{\mathrm{g}}$. The over-current protection of the DG units or at the PCC may activate. Fig. 7(b) shows some variations in the terminal voltages of the units just after the switching. The reason is that in this case, there is no synchronisation and, thus, hard switching occurs.

b) With synchronisation procedure: In this case, the phase angle, frequency and rms voltage of $v_{\mathrm{g}, 1}$ and $v_{\mathrm{util}}$ are synchronised. The obtained results are depicted in Fig. 8. Fig. 8.d) shows synchronisation of the full waveform.

Note the different scale in the vertical axes of Figs. 8(a-c) compared to the previous case. The synchronisation transient is significantly lower than in the case without synchronisation. As the voltage in the islanded microgrid is lower than the nominal

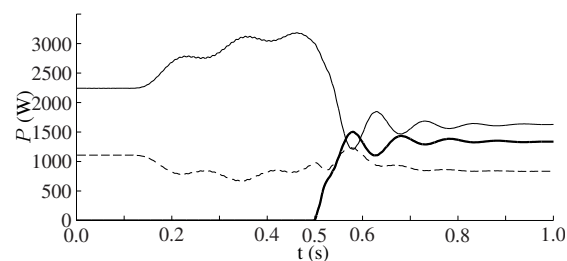

(a) Delivered active power

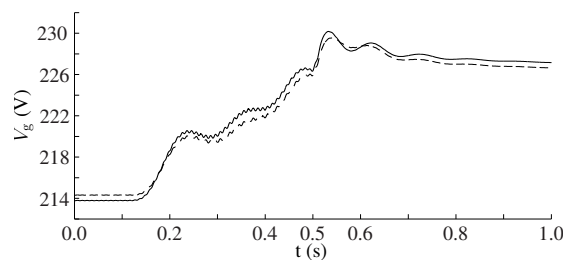

(b) Terminal rms voltage

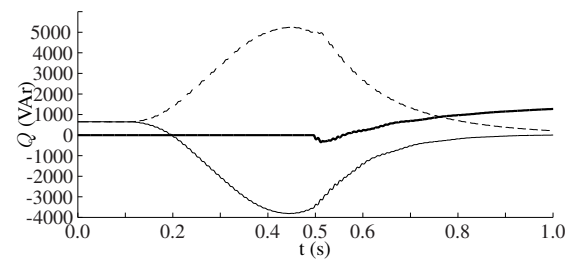

(c) Delivered reactive power

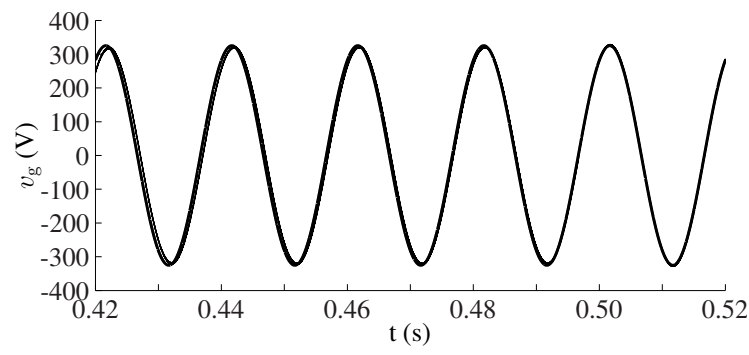

(d) Terminal voltage

Fig. 8. Basic microgrid: islanded to grid-connected, with synchronisation $(-=$ DG $1 ;----=$ DG $2 ;-=$ utility network)

voltage, the power delivered by the dispatchable DG units is significantly larger than the nominal value, i.e., a large load burden. Therefore, in the grid-connected mode, the utility network injects power into the microgrid, forcing the voltage in the network and active power of the DG units closer to the nominal values.

During synchronisation, the synchronising DG unit DG 1 delivers a significant amount of $Q$ to synchronise its voltage phase angle with the PCC voltage. This is due to the large phase angle difference chosen in this case and the small $T_{\text {sync. The }}$ other DG unit changes its $Q$ to maintain the balance. The active power of DG 1 increases during synchronisation to match its voltage with that of the PCC. In the basic microgrid, a large load, low $P_{\text {nom }}$, no secondary control to change the set points and a large initial phase angle difference are chosen such that before synchronisation the difference in $v_{\mathrm{dg}, 1}$ and $v_{\mathrm{pcc}}$ is large. In this way, the synchronisation procedure can be studied in an extreme case.

\section{B. Extended microgrid}

The microgrid configuration is depicted in Fig. 9 and the parameters are summarized in Table I. The mode transition takes 
place at the zero crossing of the voltage just before $0.6 \mathrm{~s}$. The microgrid consists of three constant-power loads with powerfactor 0.95 . The first load $P_{\text {load, } 1}$ equals $500 \mathrm{~W}, 150 \mathrm{VAr}$. The second load consumes $1 \mathrm{~kW}, 325 \mathrm{VAr}$ and the third is a $2 \mathrm{~kW}$, $650 \mathrm{VAr}$ load. Also, two RL loads with the same power-factor are included. Further, the microgrid consists of three DG units. DG 1 and DG 2 are dispatchable, DG 3 is a unit with a wide constantpower band, representing a less dispatchable DG unit. The utility network is modelled as a strong network, with $230 \mathrm{~V}$ and $50 \mathrm{~Hz}$ and is connected to the microgrid through a line impedance. The transition from islanded to grid-connected mode is studied.

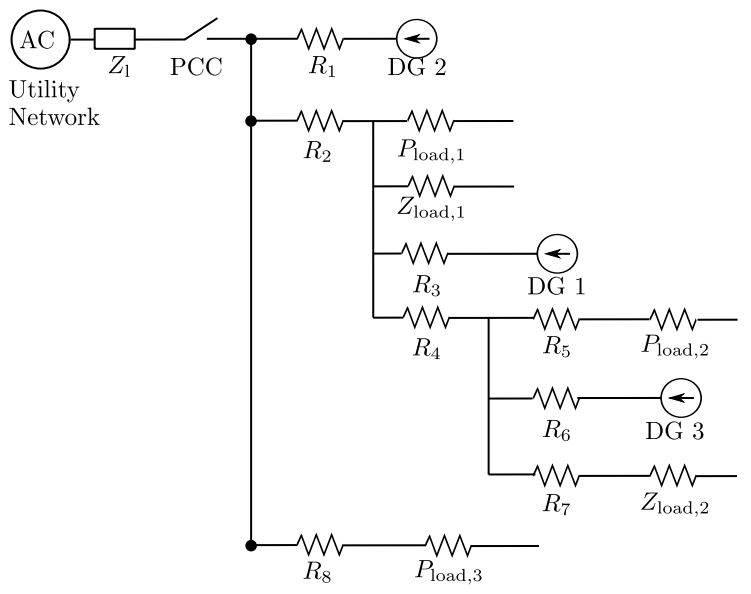

Fig. 9. Extended microgrid configuration

TABLE I

MiCROGRID CASE: PARAMETERS

\begin{tabular}{c|c||c|c} 
Parameter & value & Parameter & value \\
\hline$C_{\mathrm{dc}}$ & $1.5 \mathrm{mF}$ & $P_{\text {nom }, 3}$ & $1.55 \mathrm{~kW}$ \\
$V_{\mathrm{dc}, \text { nom }}$ & $450 \mathrm{~V}$ & $R_{1}, R_{3}, R_{6}$ & $0.5 \Omega$ \\
$V_{\mathrm{g}, \text { ref }}$ & $230 \mathrm{~V}$ & $R_{2}, R_{4}, R_{5}, R_{7}, R_{8}$ & $0.3 \Omega$ \\
$f_{\text {nom }}$ & $50 \mathrm{~Hz}$ & $Z_{\text {load }, 1}$ & $50+16 j \Omega$ \\
$P_{\text {nom }, 1}$ & $1.25 \mathrm{~kW}$ & $Z_{\text {load }, 2}$ & $75+25 j \Omega$ \\
$P_{\text {nom }, 2}$ & $1.60 \mathrm{~kW}$ & $Z_{1}$ & $0.5+0.5 \mathrm{j} \Omega$
\end{tabular}

1) Without synchronisation: First, no synchronisation procedure is performed before connecting the microgrid to the utility network. The simulation results are depicted in Fig. 10 Clearly, the mode transfer transients are unacceptable and the protection devices may turn on.

2) With synchronisation: Second, a synchronisation procedure in DG 1 is performed, with $T_{\text {sync }}=0.3 \mathrm{~s}$. The simulation results are depicted in Fig. 11. The voltages remain in the $10 \%$ limits in both modes. Fig. 11(d) shows that synchronisation of the PCC voltage and the voltage of DG 1 is achieved at the switching instant.

The synchronisation procedure leads to a significant reduction of transient voltages and currents $\left(Q, P\right.$ and $\left.V_{\mathrm{g}}\right)$ during the mode transfer. Note the different scales of the vertical axes of Figs. 10 and 11. In the islanded mode, the terminal voltages are significantly lower compared to in the grid-connected mode. The reason is that here, a large load burden is combined with a low total nominal power of the DG units. In this way, the operation in grid-connected and islanded mode differ significantly. The third

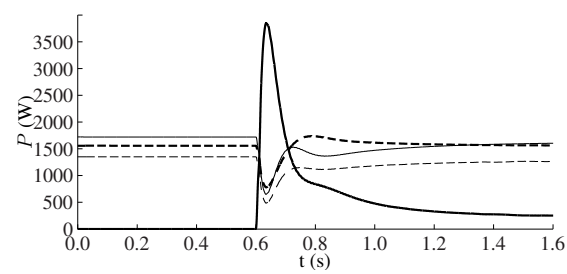

(a) Delivered active power

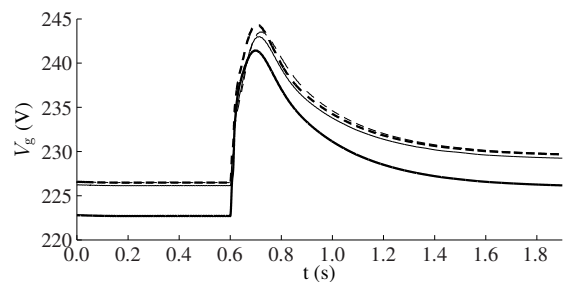

(b) Terminal rms voltage

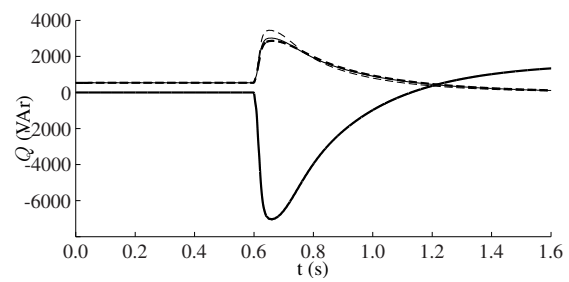

(c) Delivered reactive power

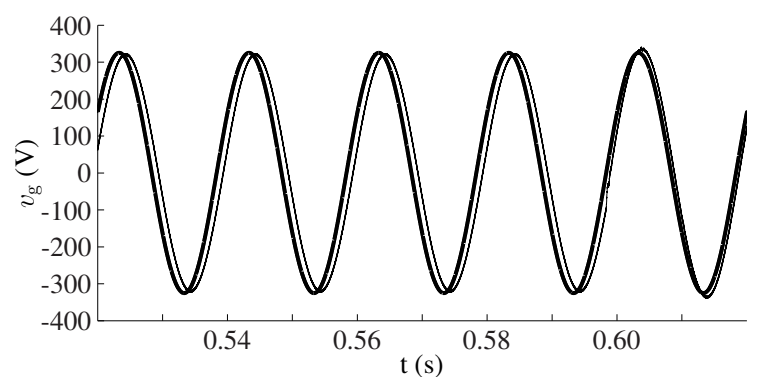

(d) Terminal voltage

Fig. 10. Extended microgrid: islanded to grid-connected, without synchronisation (- = DG 1; ---- = DG 2; ---- = DG 3; - = utility network $)$

DG unit, which is little dispatchable, clearly operates at nominal power, i.e., maximum power point, in both modes. The other units share the load in islanded mode and operate at rated power in the grid-connected mode as in this case, $V_{\mathrm{g}} \approx 230 \mathrm{~V}$.

\section{CONCLUSION}

In this paper, it is shown that VBD control is possible in both modes. In the islanded microgrid, proper power sharing, balancing and voltage control are achieved. An optimized integration and capturing of the renewable energy is achieved because of the usage of constant-power bands. In the grid-connected mode, the control strategy does not need to be changed. Without need for communication, the renewables take part in the voltage control by using soft curtailment in case of extreme voltages.

Islanding does not require additional measures as the control strategy does not need to be altered. For transition from islanded to grid-connected mode, which is a planned event, communication is used to announce the mode transition. The synchronising DG unit starts a procedure to synchronise its terminal voltage $v_{\mathrm{g}}$ with $v_{\text {util }}$. This synchronisation procedure is achieved by altering the 


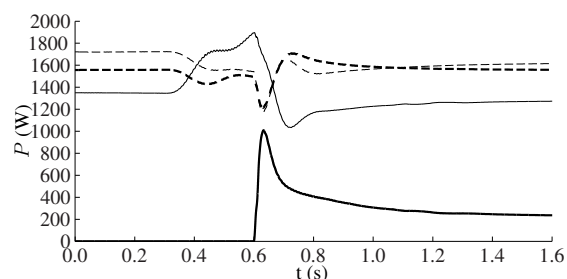

(a) Delivered active power

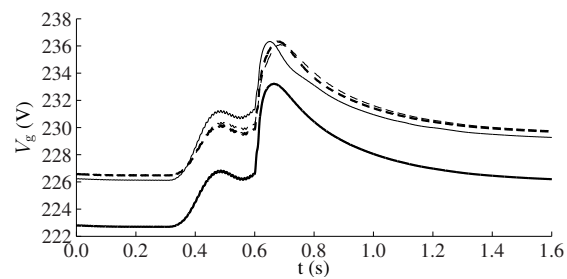

(b) Terminal rms voltage

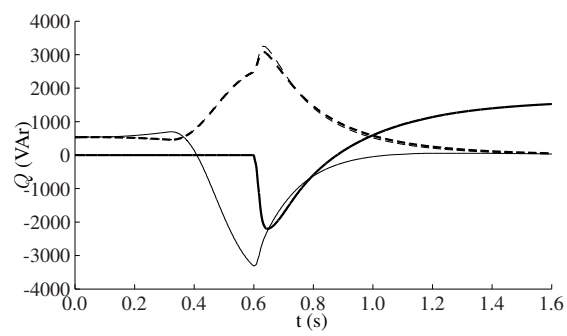

(c) Delivered reactive power

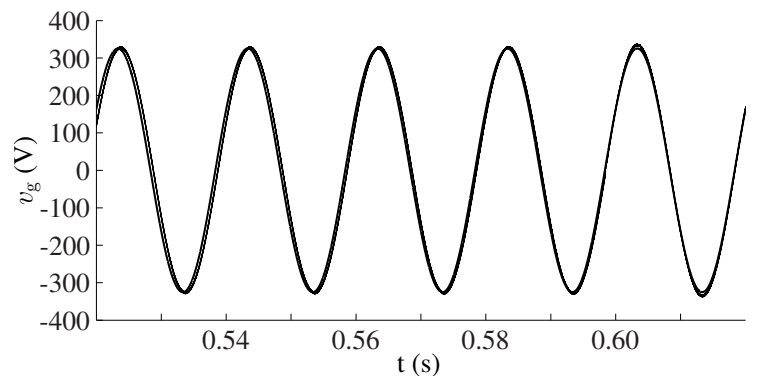

(d) Terminal voltage

Fig. 11. Extended microgrid: islanded to grid-connected, with synchronisation $(-=$ DG $1 ;----=$ DG $2 ;----=$ DG $3 ;-=$ utility network $)$

VBD control with an additional term in the $P_{\mathrm{dc}} / V_{\mathrm{g}}$ and $Q / f$ controllers. These controllers synchronise the rms voltage and the frequency/phase angle respectively. Hence, in this paper, a smooth mode-transfer is achieved by modifying the VBD control strategy with a synchronisation procedure to connect the islanded microgrid with the utility.

\section{REFERENCES}

[1] P. Dondi, D. Bayoumi, C. Haederli, D. Julian, and M. Suter, "Network integration of distributed power generation," Journal of Power Sources, vol. 106, pp. 1-9, 2002.

[2] I. Wasiak, M. C. Thoma, C. E. T. Foote, R. Mienski, R. Pawelek, P. Gburczyk, and G. M. Burt, "A power-quality management algorithm for lowvoltage grids with distributed resources," vol. 23, no. 2, pp. 1055-1062, Apr. 2008

[3] Y. Li, D. M. Vilathgamuwa, and P. C. Loh, "Microgrid power quality enhancement using a three-phase four-wire grid-interfacing compensator," vol. 41, no. 6, pp. 1707-1719, Nov./Dec 2005.

[4] S. Barsali, M. Ceraolo, P. Pelacchi, and D. Poli, "Control techniques of dispersed generators to improve the continuity of electricity supply," in Proc. IEEE PES Winter Meeting, 2002, pp. 789-794.
[5] M. H. J. Bollen and N. Etherden, "Overload and overvoltage in low-voltage and medium-voltage networks due to renewable energy - some illustrative case studies," in IEEE PES Innovative Smart Grid Technologies (ISGT 2011), Manchester, UK, Dec. 5-7, 2011.

[6] R. H. Lasseter, A. Akhil, C. Marnay, J. Stephens, J. Dagle, R. Guttromson, A. Meliopoulous, R. Yinger, and J. Eto, "The CERTS microgrid concept, white paper on integration of distributed energy resources," in California Energy Commission, Office of Power Technologies - U.S. Department of Energy, LBNL-50829, http://certs.lbl.gov, Apr. 2002.

[7] R. H. Lasseter and P. Paigi, "Microgrid: A conceptual solution," in Proc. IEEE Power Electron. Spec. Conf. (PESC 2004), Aachen, Germany, 2004.

[8] N. Lidula and A. D. Rajapakse, "Microgrids research: A review of experimental microgrids and test systems," Renewable and Sustainable Energy Reviews, vol. 15, no. 1, pp. 186-202, 2011.

[9] A. Mehrizi-Sani and R. Iravani, "Potential-function based control of a microgrid in islanded and grid-connected modes," vol. 25 , no. 4, pp. $1883-$ 1891, Nov. 2010.

[10] H. Farhangi, "The path of the smart grid," in IEEE Power \& Energy Magazine, Jan./Feb. 2010, vol. 8, no. 1, pp. 18-28.

[11] L. R. Limongi, R. Bojoi, G. Griva, and A. Tenconi, "Digital current-control schemes," IEEE Ind. Electr. Magazine, vol. 3, no. 1, pp. 20-31, Mar. 2009.

[12] M. P. Kazmierkowski and L. Malesani, "Current control techniques for three-phase voltage-source PWM converters: A survey," vol. 45, no. 5, pp. 691-703, Oct. 1998.

[13] B. Meersman, B. Renders, L. Degroote, T. Vandoorn, and L. Vandevelde, "Three-phase inverter-connected DG-units and voltage unbalance," Electric Power Systems Research, vol. 81, no. 4, pp. 899-906, 2011.

[14] M. C. Chandorkar, D. M. Divan, and R. Adapa, "Control of parallel connected inverters in standalone ac supply systems," vol. 29, no. 1, pp. 136-143, Jan./Feb. 1993.

[15] J. M. Guerrero, J. Matas, L. García de Vicuña, M. Castilla, and J. Miret, "Wireless-control strategy for parallel operation of distributed-generation inverters," vol. 53, no. 5, pp. 1461-1470, Oct. 2006.

[16] J. A. Peças Lopes, C. L. Moreira, and A. G. Madureira, "Defining control strategies for microgrids in islanded operation," vol. 21, no. 2, pp. 916-924, 2006.

[17] H. Laaksonen, P. Saari, and R. Komulainen, "Voltage and frequency control of inverter based weak LV network microgrid," in 2005 International Conference on Future Power Systems, Amsterdam, Nov. 18, 2005.

[18] A. Engler, O. Osika, M. Barnes, and N. Hatziargyriou, DB2 Evaluation of the local controller strategies. www.microgrids.eu/micro2000, Jan. 2005.

[19] T. L. Vandoorn, B. Meersman, L. Degroote, B. Renders, and L. Vandevelde, "A control strategy for islanded microgrids with dc-link voltage control," vol. 26, no. 2, pp. 703-713, Apr. 2011.

[20] T. L. Vandoorn, B. Renders, L. Degroote, B. Meersman, and L. Vandevelde, "Active load control in islanded microgrids based on the grid voltage," IEEE Trans. on Smart Grid, vol. 2, no. 1, pp. 139-151, Mar. 2011.

[21] Z. Yao, L. Xiao, and Y. Yan, "Seamless transfer of single-phase gridinteractive inverters between grid-connected and stand-alone modes," vol. 25 , no. 6, pp. 1597-1603, Jun. 2010.

[22] Y. Li, D. M. Vilathgamuwa, and P. C. Loh, "Design, analysis, and real-time testing of a controller for multibus microgrid system," vol. 19, no. 5, pp. 1195-1204, Sep. 2004.

[23] J. C. Vasquez, J. M. Guerrero, A. Luna, P. Rodriguez, and R. Teodorescu, "Adaptive droop control applied to voltage-source inverters operating in grid-connected and islanded modes," vol. 56, no. 10, pp. 4088-4096, Oct. 2009.

[24] J. Kim, J. M. Guerrero, P. Rodriguez, R. Theodorescu, and K. Nam, "Mode adaptive droop control with virtual output impedances for inverter-based flexible AC microgrid," vol. 26, no. 3, pp. 689-701, Mar. 2011.

[25] J. M. Guerrero, J. C. Vásquez, J. Matas, L. García de Vicuña, and M. Castilla, "Hierarchical control of droop-controlled AC and DC microgrids - A general approach towards standardization," vol. 58, no. 1, pp. 158-172, Jan. 2011

[26] T. L. Vandoorn, C. Ionescu, J. D. M. D. Kooning, R. D. Keyser, and L. Vandevelde, "Theoretical analysis and experimental validation of singlephase direct vs. cascade voltage control in islanded microgrids," vol. 60, no. 2, pp. 789-798, feb 2013.

[27] A. Engler, "Applicability of droops in low voltage grids," DER Journal, no. 1, Jan. 2005.

[28] A. Yafaoui, B. Wu, and R. Cheung, "Implementation of maximum power point tracking algorithm for residential photovoltaic systems," in in the 2nd Canadian Solar Buildings Conference, Calgary, June 10-14, 2007.

[29] D. P. Hohm and M. E. Ropp, "Comparative study of maximum power point tracking algorithms," Progress in Photovoltaics: Research and Applications, vol. 11, pp. 47-62, 2003.

[30] V. Salas, E. Olias, A. Barrado, and A. Lazaro, "Review of the maximum power point tracking algorithms for stand-alone photovoltaic systems," Solar Energy Materials and Solar Cells, vol. 90, pp. 1555-1578, 2006. 


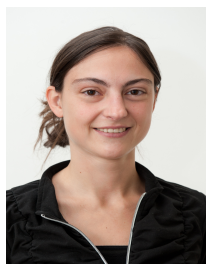

Tine Vandoorn (S'09) was born in Torhout, Belgium in 1985. She received the M.S. in electromechanical engineering from Ghent University, Ghent, Belgium, in 2008. In 2008, she joined the Electrical Energy Laboratory (EELAB) of Ghent University where she is currently pursuing the Ph.D. degree in electromechanical engineering. Her present research interests include electric power systems, voltage and power control of DG units, management of microgrids and smart microgrids. In 2009, she was awarded a grant as Ph.D. fellow of the Research Foundation - Flanders (FWO).

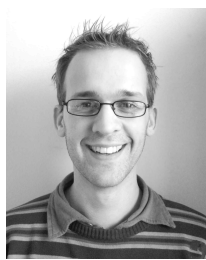

Bart Meersman (S'07) was born in Sint-Niklaas, Belgium in 1983. He received the M.S. and Ph.D degree in electromechanical engineering from Ghent University, Belgium, in 2006 and 2012, respectively. He is with the Electrical Energy Laboratory (EELAB), Ghent University, since 2006, where he is a postdoctoral researcher in electrical power engineering. His research activities are in the field of electric power systems, renewable energy applications, power electronics and digital control of power electronic converters and their contribution to power quality.

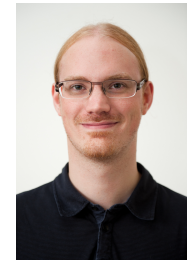

Jeroen De Kooning (S'09) was born in Kapellen, Belgium in 1987. He received the M.S. degree in electromechanical engineering from Ghent University, Belgium, in 2010. Since then, he is with the Electrical Energy Laboratory (EELAB) of Ghent University and is currently pursuing the $\mathrm{Ph} . \mathrm{D}$. degree in electromechanical engineering. His present research interests include wind energy systems, control of power-electronic converters and brush-less ac drives.

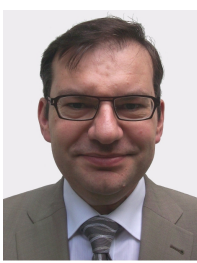

Lieven Vandevelde (M05 - SM07) was born in Eeklo, Belgium, in 1968. He graduated as Master of Electromechanical Engineering and received the Ph.D. degree in electromechanical engineering from Ghent University, Belgium, in 1992 and 1997, respectively. He is with the Electrical Energy Laboratory (EELAB), Ghent University, where he has been a professor in electrical power engineering since 2004 . His research and teaching activities are in the field of electric power systems, electrical machines and (computational) electromagnetics. 